\title{
Cine DENSE MRI of mechanical activation in heart failure patients referred for cardiac resynchronization therapy
}

\author{
Daniel A Auger ${ }^{1 *}$, Sophia X Cui ${ }^{1}$, Xiao Chen² ${ }^{2}$ Jorge A Gonzalez ${ }^{3}$, Christopher M Kramer ${ }^{4,3}$, Kenneth C Bilchick ${ }^{3}$, \\ Frederick H Epstein ${ }^{1,4}$ \\ From 19th Annual SCMR Scientific Sessions \\ Los Angeles, CA, USA. 27-30 January 2016
}

\section{Background}

In patients with heart failure (HF) and left bundle branch block (LBBB) cardiac resynchronization therapy (CRT) improves left ventricular (LV) function and leads to reverse LV remodeling. A major concern with CRT remains that using current clinical indicators and implementation methods, $30-40 \%$ of patients are nonresponders. CRT response is highly dependent on the presence of delayed mechanical activation and absence of scar at the LV lead implantation site [1]. The aims of this study were to establish the correlation between mechanical activation time measured by cine DENSE MRI and electrical activation time (QLV) at the LV lead implantation site, and to characterize the heterogeneity of the location of the site of the latest mechanically activated segment in patients with HF-LBBB. Furthermore, we evaluated the frequency in which CRT leads are placed in suboptimal regions.

\section{Methods}

Short axis images were acquired on a $1.5 \mathrm{~T}$ MR system from 6 healthy subjects and 50 HF-LBBB patients (27 with myocardial scar and 23 without scar) with established clinical criteria for CRT. Late gadolinium enhancement (LGE) was used to detect the scar location. DENSE circumferential strain was computed using previously described methods [2,3]. Spatiotemporal strain data were arranged into a $2 \mathrm{D}$ matrix over space (LV segment) and time (cardiac phase) and singular value decomposition (SVD) was used to denoise the strain data. An active contour method automatically detected regions of delayed mechanical activation, defined by the time-of-onset of contraction, as per Figure 1(A). Mechanical activation time was correlated with QLV which was recorded during the CRT procedure.

\section{Results}

Figure 1(B, C) shows good correlations between DENSE mechanical activation time and QLV at matched locations in the LV for patients without and with scar, with a greater electrical-mechanical delay in regions with scar Figure $1(\mathrm{C})$. Figure 2(A, B) illustrates the variation in the location of the latest mechanically activated segment in 2 patients with HF-LBBB, and the overall heterogeneity of the site of the latest mechanically activated segment is shown in a bulls-eye plot in Figure 2(C) for all patients. Using standard CRT methods, in $55 \%$ of patients the LV lead was placed in an area with less than $80 \%$ of the maximal delay, and in $30 \%$ of patients the LV lead was placed in a region with less than $60 \%$ of the maximal mechanical delay.

\section{Conclusions}

In HF patients selected for CRT, mechanical activation times measured with cine DENSE correlate well with electrical activation times. Imaging of mechanical activation demonstrates heterogeneity of the site of latest activation. Based on the heterogeneity and the suboptimal lead locations achieved using current CRT methods, pre-procedure MRI of mechanical activation shows potential for improving the implementation of CRT. 

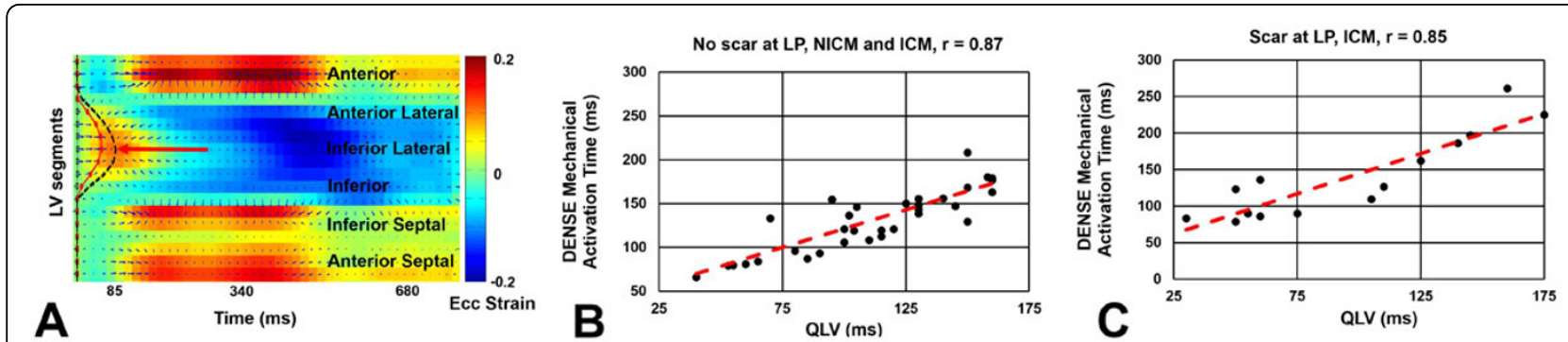

Figure 1 (A) An active contour applied to a strain matrix automatically depicts the region of late mechanical activation (red arrow). (B) Correlation plot between DENSE mechanical activation time and electrical activation time (QLV) for segments where the CRT LV lead was placed in a region without scar. (C) Correlation plot between DENSE mechanical activation time and QLV for segments where the CRT LV lead was placed in a region with scar

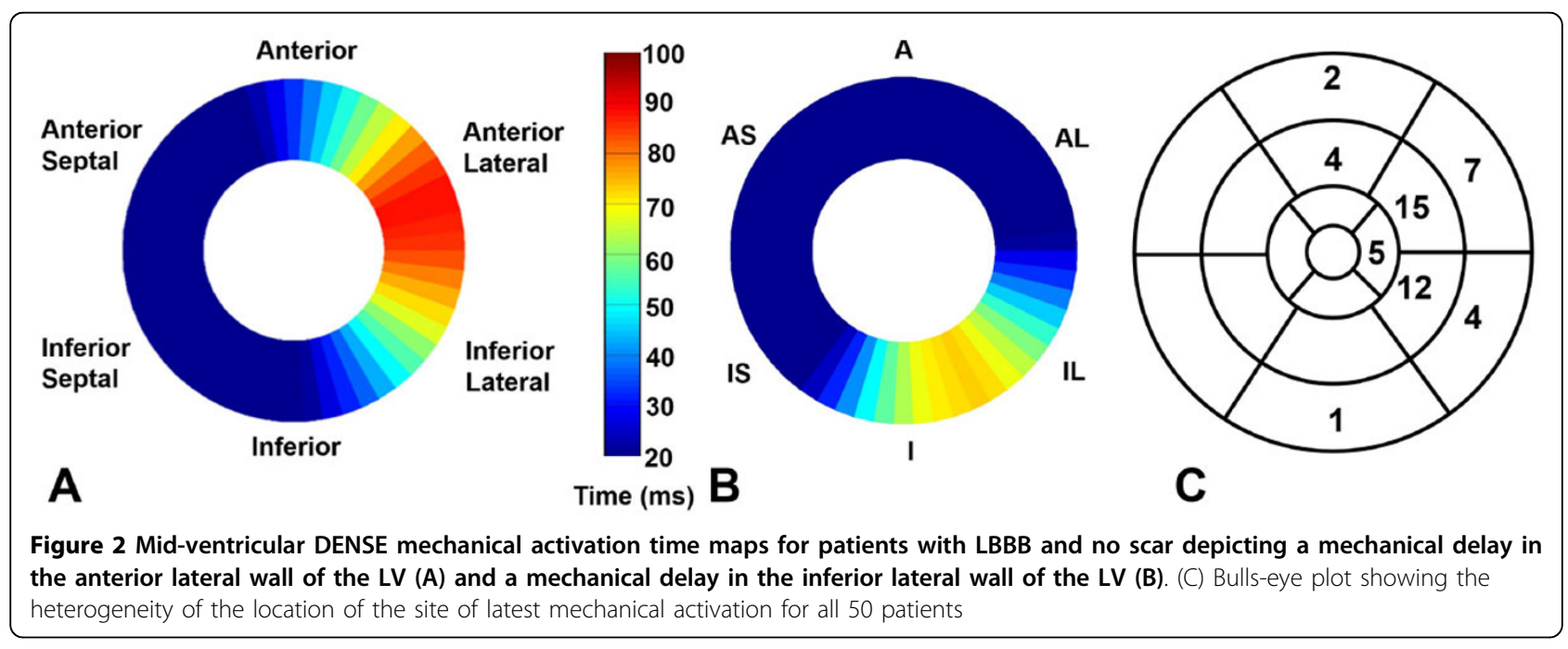

\section{Authors' details}

'Biomedical Engineering, University of Virginia, Charlottesville, VA, USA.

${ }^{2}$ Medical Solutions, Siemens, Princeton, NJ, USA. ${ }^{3}$ Medicine, Cardiovascular

Medicine, University of Virginia, Charlottesville, VA, USA. ${ }^{4}$ Radiology and

Medical Imaging, University of Virginia, Charlottesville, VA, USA.

Published: 27 January 2016

\section{References}

1. Bilchick, et al: JACC 2014, 63:1657-66.

2. Spottiswoode, et al: IEEE TMI 2007, 26:15-30

3. Spottiswoode, et al: MIA 2009, 13:105-115.

doi:10.1186/1532-429X-18-S1-P215

Cite this article as: Auger et al: Cine DENSE MRI of mechanical activation in heart failure patients referred for cardiac resynchronization therapy. Journal of Cardiovascular Magnetic Resonance 2016 18(Suppl 1): P215.
Submit your next manuscript to BioMed Central and take full advantage of:

- Convenient online submission

- Thorough peer review

- No space constraints or color figure charges

- Immediate publication on acceptance

- Inclusion in PubMed, CAS, Scopus and Google Scholar

- Research which is freely available for redistribution 